\title{
On a certain nonlocal problem for a heat equation
}

\author{
Olga Danilkina \\ Department of Mathematics, School of Natural and Mathematical Sciences, \\ Dodoma University, P.O.BOX 259, Dodoma, Tanzania \\ Email: olga.danilkina@gmail.com
}

Copyright (C)2014 Olga Danilkina. This is an open access article distributed under the Creative Commons Attribution License, which permits unrestricted use, distribution, and reproduction in any medium, provided the original work is properly cited.

\begin{abstract}
The aim of this paper is to study the nonlocal problem with the integral condition of the first kind for the heat equation. We prove existense and uniqueness of a generalized solution for this problem. The proof is based on the obtained apriory estimate and Galerkin method.
\end{abstract}

Keywords: energy inequality, Galerkin method, heat equation, nonlocal.

\section{Introduction}

Over the previous years, various phenomena of modern natural science were successfully described in terms of nonlocal mathematical models and nonlocal problems for evolution equations have been studied actively. Problems with nonlocal integral conditions form an important class of nonlocal problems. The first papers devoted to secondorder partial differential equations with nonlocal integral conditions go back to Cannon [1] and Kamynin [4]. Here are some recent works [11], [12], [13]. See also references therein. Nonlocal boundary value problems with the second kind integral conditions for parabolic equations have been studied in [3], [14], [5].

In this article, we consider the problem for the equation

$u_{t}=u_{x x}+c(t) u+f(x, t), \quad(x, t) \in Q_{T}$,

with the initial and nonlocal boundary conditions

$u(x, 0)=\varphi(x)$

$u_{x}(0, t)=0$

$\int_{0}^{l} K(x) u(x, t) d x=E(t)$

where $Q_{T}=\{(x, t): 0<x<l, 0<t<T\}$.

Note that the integral condition (4) has the form of the first kind operator equation which is incorrect and can be solved with sufficient restrictions on the kernel and right-hand side part [2]. For the second kind nonlocal integral condition, e.g. $u(l, t)+\int_{0}^{l} K(x) u(x, t) d x=g(t)$, such problems do not appear as we can introduce the operator 
$B u=\int_{0}^{l} K(x) u(x, t) d x$ and consider the inegral equation of the second kind $u(x, t)+\int_{0}^{l} K(\xi) u(\xi, t) d \xi=G(x, t)$, where $G(l, t)=g(x)$. This technique has been applied in [6], [7] for a multidimensional hyperbolic equation.

Motivated by the above works, we consider the solvability of the nonlocal problem (1)-(4) with the inegral condition in the form of the first kind integral operator. It is shown that in case of a single space variable we can avoid difficulties caused by the condition (4).

\section{Preliminary notes}

Lemma 2.1 Let $c(t) \in C[0, T] ; f(x, t) \in L_{2}\left(Q_{T}\right) ; \varphi(x) \in W_{2}^{1}(0, l) ; K(x) \in C^{2}[0, l], K(l) \neq 0 ; E(t) \in C^{1}[0, T]$ and

$E(0)=\int_{0}^{l} K(x) \varphi(x) d x$.

Then the problem (1)-(4) is equivalent to the problem

$u_{t}=u_{x x}+c(t) u+f(x, t)$

$u(x, 0)=\varphi(x), \quad u_{x}(0, t)=0$,

$u_{x}(l, t)=\int_{0}^{l} H(x) u_{x}(x, t) d x+F(t)$

where $H(x)=\frac{K_{x}(x)}{K(l)}, F(t)=\frac{E^{\prime}(t)-c(t) E(t)-f_{1}}{K(l)}, f_{1}(t)=\int_{0}^{l} K f d x$.

Proof. Let $u(x, t)$ be the solution of the problem (1)-(4). Then (1) implies that

$\int_{0}^{l} K(x) u_{t}(x, t) d x=\int_{0}^{l} K(x) u_{x x}(x, t) d x+\int_{0}^{l} K(x) c(t) u(x, t) d x+\int_{0}^{l} K(x) f(x, t) d x$.

In view of the condition (4) and identity (7) we obtain

$E^{\prime}(t)=K(l) u_{x}(l, t)+c(t) E(t)+f_{1}(t)-\int_{0}^{l} K_{x} u_{x}(x, t) d x$.

And hence, $\quad u_{x}(l, t)=\frac{1}{K(l)}\left(\int_{0}^{l} K_{x}(x) u_{x}(x, t) d x+E^{\prime}(t)-c(t) E(t)-f_{1}\right)$.

Now, consider the problem (1)-(3), (6). The condition (8) follows from (6) and then

$E^{\prime}(t)=\int_{0}^{l} K(x) u_{x x} d x+c(t) E(t)+\int_{0}^{l} K(x) f(x, t) d x$.

As (7) holds we obtain

$E^{\prime}(t)=\int_{0}^{l} K(x) u_{t} d x-\int_{0}^{l} K(x) c(t) u d x-\int_{0}^{l} K(x) f(x, t) d x+c(t) E(t)+\int_{0}^{l} K(x) f(x, t) d x$.

Therefore, $\frac{d}{d t}\left(E(t)-\int_{0}^{l} K(x) u d x\right)=c(t)\left(E(t)-\int_{0}^{l} K(x) c(t) u d x\right)$.

Denote $v(t)=E(t)-\int_{0}^{l} K(x) u d x$. Then the function $v(t)$ is a solution of the following Cauchy problem $v^{\prime}(t)=c(t) v(t), v(0)=0$.

Under the conditions on the coefficient $c(t)$ this problem has a unique solution $v=0$ [10], and hence, 
$E(t)-\int_{0}^{l} K(x) u d x=0$ and $\int_{0}^{l} K(x) u(x, t) d x=E(t)$. This ends the proof.

We shall study the problem (1)- (3), (6).

Let $W_{2}^{1}\left(Q_{T}\right)$ be the usual Sobolev space. We shall define

$$
\widehat{W}_{2}^{1}\left(Q_{T}\right)=\left\{v: v \in W_{2}^{1}\left(Q_{T}\right), v(x, T)=0\right\} .
$$

First we give a definition of a generalized solution of the problem using the standard method [9]. We assume that $u(x, t)$ is a classical solution of the problem (1)-(3), (6), multiply (1) by $\eta \in \widehat{W}_{2}^{1}\left(Q_{T}\right)$ and integrate over $Q_{T}$. It follows from (2), (3), (6) and integration by parts that

$\int_{Q_{T}}\left(-u \eta_{t}+u_{x} \eta_{x}-c u \eta\right) d x d t=\int_{Q_{T}} f \eta d x d t+\int_{0}^{l} \varphi(x) \eta(x, 0) d x+\int_{0}^{T}\left(\int_{0}^{l} H(x) u_{x}(x, t) d x+F(t)\right) \eta(l, t) d t$.

Definition 2.2 A function $u(x, t) \in W_{2}^{1,0}\left(Q_{T}\right)$ is said to be a generalized solution of the problem (1) - (3), (6) if for every $\eta(x, t) \in \widehat{W}_{2}^{1}\left(Q_{T}\right)$ the identity (9) holds.

\section{Main result}

Theorem 3.1 Let $c(t) \in C[0, T] ; f(x, t) \in L_{2}\left(Q_{T}\right) ; \varphi(x) \in W_{2}^{1}(0, l) ; K(x) \in C^{2}[0, l], K(l) \neq 0 ; E(t) \in C^{1}[0, T]$, $\int_{0}^{l} K_{x x}^{2}(x) d x \neq 0, K_{x}(0)=0$ and $E(0)=\int_{0}^{l} K(x) \varphi(x) d x$. Then there exists a unique generalized solution of the problem (1)-(3), (6).

Proof. First we prove the uniqueness. To this end we obtain several inequalities and then use Gronwall's lemma. To prove the existence we construct approximations of the generalized solution by Galerkin method and obtain a priori estimates to guarantee convergence of approximations. Finally, we show that the limit of approximations is the required solution.

1. Uniqueness. Assume that there exist two different solutions $u_{1}(x, t)$ and $u_{2}(x, t)$ of the problem (1)-(3), (6). Then $u(x, t)=u_{1}(x, t)-u_{2}(x, t)$ is an element of $W_{2}^{1,0}\left(Q_{T}\right)$ and the identity

$\int_{Q_{T}}\left(-u \eta_{t}+u_{x} \eta_{x}-c u \eta\right) d x d t=\int_{0}^{T}\left(\int_{0}^{l} H(x) u_{x}(x, t) d x\right) \eta(l, t) d t$

holds for every $\eta(x, t) \in \widehat{W}_{2}^{1}\left(Q_{T}\right)$. It follows from integration by parts and the condition $H(0)=0$ that

$\int_{Q_{T}}\left(-u \eta_{t}+u_{x} \eta_{x}-c u \eta\right) d x d t=\int_{0}^{T}\left(H(l) u(l, t)-\int_{0}^{l} H_{x}(x) u(x, t) d x\right) \eta(l, t) d t$.

For an arbitrary $b \in[0, T]$, take $\eta$ as

$\eta(x, t)=\left\{\begin{array}{cl}0, & t \in[b, T], \\ \int_{b}^{t} u(x, \tau) d \tau, & t \in[0, b],\end{array}\right.$

It is easy to see that $\eta(x, t) \in W_{2}^{1}\left(Q_{T}\right), \eta(x, T)=0$ and $\eta_{x t}=u_{x} \in L_{2}\left(Q_{T}\right)$.

Substitute $\eta(x, t)$ from (11) in (10) and express $u$ in terms of $\eta$ and its derivatives. As a result we obtain the equality

$\int_{0}^{b} \int_{0}^{l}\left(-\eta_{t}^{2}+\eta_{t x} \eta_{x}-c \eta_{t} \eta\right) d x d t=\int_{0}^{b}\left(H(l) \eta_{t}(l, t)-\int_{0}^{l} H_{x} \eta_{t} d x\right) \eta(l, t) d t$.

It follows from integration by parts in the second term in the left-habd side and conditions $\eta(x, b)=0, \eta_{x}(x, b)=$ 0 that

$-\int_{0}^{b} \int_{0}^{l} \eta_{t}^{2} d x d t-\frac{1}{2} \int_{0}^{l} \eta_{x}^{2}(x, 0) d x-\int_{0}^{b} \int_{0}^{l} c \eta_{t} \eta d x d t=\int_{0}^{b} \eta(l, t)\left(H(l) \eta_{t}(l, t)-\int_{0}^{l} H_{x} \eta_{t} d x\right) d t$. 
Since $\int_{0}^{b} H(l) \eta(l, t) \eta_{t}(l, t) d t=-\frac{1}{2} H(l) \eta^{2}(l, 0)$, so

$\int_{Q_{b}} \eta_{t}^{2} d x d t+\frac{1}{2} \int_{0}^{l} \eta_{x}^{2}(x, 0) d x=-\int_{Q_{b}} c \eta_{t} \eta d x d t+\frac{1}{2} H(l) \eta^{2}(l, 0)+\int_{0}^{b} \eta(l, t) \int_{0}^{l} H_{x} \eta_{t} d x d t$.

Our next aim is to derive an estimate of a right-hand side of (13). Taking into account hypotheses of the theorem we can see that there exists positive number $c_{1}$ such that $\max |c(t)| \leq c_{1}$. Applying the Cauchy inequality we obtain

$\left|\int_{Q_{b}} c \eta_{t} \eta d x d t\right| \leq c_{1}^{2} \int_{Q_{b}} \eta^{2} d x d t+\frac{1}{4} \int_{Q_{b}} \eta_{t}^{2} d x d t$

$\left|\int_{0}^{b} \eta(l, t) \int_{0}^{l} H_{x} \eta_{t} d x d t\right| \leq \int_{0}^{b}|\eta(l, t)|\left|\int_{0}^{l} H_{x} \eta_{t} d x\right| d t \leq H_{1} \int_{0}^{b} \eta^{2}(l, t) d t+\frac{1}{4 H_{1}} \int_{0}^{b}\left(\int_{0}^{l} H_{x} \eta_{t} d x\right)^{2} d t$

where $H_{1}=\int_{0}^{l} H_{x}^{2} d x$. Based on [8], we note that for any $u \in W_{2}^{1,0}\left(Q_{T}\right)$ the following inequality holds

$u^{2}(l, t) \leq \int_{0}^{l}\left(\varepsilon u^{2}(x, t)+a(\varepsilon) u_{x}^{2}(x, t)\right) d x$,

where $\varepsilon$ is and arbitrary positive constant. As $\eta(x, t) \in W_{2}^{1}\left(Q_{T}\right)$ then from (15) it follows that

$\frac{1}{2} H(l) \eta^{2}(l, 0) \leq \int_{0}^{l}\left(\frac{1}{4} \eta_{x}^{2}(x, 0)+a(\rho) \eta^{2}(x, 0)\right) d x$

and

$\left|\int_{0}^{b} \eta(l, t) \int_{0}^{l} H_{x} \eta_{t} d x d t\right| \leq H_{1} \int_{0}^{b} \int_{0}^{l}\left(\beta \eta_{x}^{2}(x, t)+a(\beta) \eta^{2}(x, t)\right) d x d t+\frac{1}{4} \int_{Q_{b}} \eta_{t}^{2} d x d t$,

Appling the estimates (14)-(16) to (13) we obtain

$\frac{1}{2} \int_{Q_{b}} \eta_{t}^{2} d x d t+\frac{1}{4} \int_{0}^{l} \eta_{x}^{2}(x, 0) d x \leq\left(c_{1}^{2}+H_{0} a(\beta)\right) \int_{Q_{b}} \eta^{2} d x d t++\frac{1}{2} H(l) a(\rho) \int_{0}^{l} \eta^{2}(x, 0) d x+\int_{Q_{b}} \eta_{x}^{2}(x, t) d x d t$.

It easy to see that $\eta^{2}(x, t) \leq b \int_{0}^{b} \eta_{t}^{2}(x, \tau) d \tau, \int_{0}^{l} \eta^{2}(x, 0) d x \leq b \int_{Q_{b}} \eta_{t}^{2}(x, t) d x d t$ and

$\int_{Q_{b}} \eta^{2}(x, t) d x d t \leq b^{2} \int_{Q_{b}} \eta_{t}^{2}(x, t) d x d t$. Therefore,

$\int_{Q_{b}} \eta_{t}^{2}(x, t) d x d t+\int_{0}^{l} \eta_{x}^{2}(x, 0) d x \leq 4 \int_{Q_{b}} \eta_{x}^{2}(x, t) d x d t$

where $b=\min \left\{1 / \sqrt{8 c_{2}}, 1 / 8 c_{2}\right\}, c_{2}=\min \left\{c_{1}^{2}+H_{0} a(\beta), \frac{1}{2} H(l) a(\rho)\right\}$.

Introduce the function $\int_{0}^{t} u(x, \tau) d \tau=y(x, t)$. For $t \in[0, b] \eta(x, t)=y(x, t)-y(x, b)$. And hence,

$\int_{0}^{l} y_{x}^{2}(x, b) d x \leq 4 \int_{Q_{b}}\left(y_{x}(x, t)-y_{x}(x, b)\right)^{2} d x d t \leq 8 \int_{Q_{b}}\left(y_{x}^{2}(x, t)+y_{x}^{2}(x, b)\right) d x d t=$

$=8 \int_{Q_{b}} y_{x}^{2}(x, t) d x d t+8 b \int_{0}^{l} y_{x}^{2}(x, b) d x d t$.

For $\quad b \leq 1 / 16 \quad$ we obtain $\quad \int_{0}^{l} y_{x}^{2}(x, b) d x \leq 16 \int_{Q_{b}} y_{x}^{2}(x, t) d x d t$. 
The inequality (19) is valid for all $b \in\left[0, b_{1}\right]$, where $b_{1}$ is defined as $b_{1}=\min \left\{1 / \sqrt{8 c_{2}}, 1 / 8 c_{2}, 1 / 16\right\}$. We write (19) in the form $\frac{d}{d b} \int_{0}^{b} \int_{0}^{l} y_{x}^{2}(x, t) d x d t \leq 16 \int_{0}^{b} \int_{0}^{l} y_{x}^{2}(x, t) d x d t$.

Since $y(x, 0)=0$, so $y_{x}(x, 0)=0$. Therefore, applying Gronwall's lemma we obtain $\int_{Q_{b}} y_{x}^{2}(x, t) d x d t=0$. And hence, $y_{x}(x, b) \equiv 0$ for all $b \in\left[0, b_{1}\right]$. It follows immediately that $\eta_{x}(x, t)=y_{x}(x, t)-y_{x}(x, b) \equiv 0$ for $t \in\left[0, b_{1}\right]$.

By $(18)$ we conclude that $\eta_{t}(x, t)=u(x, t)=0$ for $t \in\left[0, b_{1}\right]$. We repeat these arguments for $t \in\left[b_{1}, 2 b_{1}\right]$ and then continue this procedure. It follows that $u(x, t)=0$ for all $t \in[0, T]$. Therefore there exists at most one solution of (1)- (3), (6).

2. Existence. Let $\left\{\psi_{k}(x)\right\} \in C^{1}[0, l]$ be a basis in $W_{2}^{1}(0, l)$ and

$\left(\psi_{i}, \psi_{j}\right)_{L_{2}(0, l)}= \begin{cases}1, & i=j \\ 0, & i \neq j\end{cases}$

We define the approximations

$u^{N}(x, t)=\sum_{k=1}^{N} c_{k}^{N}(t) \psi_{k}(x)$

where $c_{k}^{N}(t)$ are solutions of the Cauchy problem

$$
\begin{aligned}
& \int_{0}^{l} u_{t}^{N}(x, t) \psi_{m}(x) d x+\int_{0}^{l} u_{x}^{N}(x, t) \psi_{m}^{\prime}(x) d x-\int_{0}^{l} c(t) u^{N}(x, t) \psi_{m}(x) d x= \\
& =\int_{0}^{l} f(x, t) \psi_{m}(x) d x+\left(\int_{0}^{l} H(x) u_{x}^{N}(x, t) d x+F(t)\right) \psi_{m}(l), \\
& c_{m}^{N}(0)=\left(\varphi, \psi_{m}\right), \quad m=\overline{1, N} .
\end{aligned}
$$

We write the Cauchy problem $(21)-(22)$ such that

$\frac{d}{d t} c_{m}^{N}(t)+\sum_{k=1}^{N} A_{k, m}(t) c_{k}^{N}(t)=\int_{0}^{l} f(x, t) \psi_{m}(x) d x-F(t) \psi_{m}(l), m=\overline{1, N}$

where $A_{k, m}(t)=\int_{0}^{l} \psi_{k}^{\prime}(x) \psi_{m}^{\prime}(x) d x-c(t)-\psi_{m}(l) \int_{0}^{l} H(x) \psi_{k}^{\prime}(x) d x$.

Under the hypothesis of the theorem coefficients $A_{k, m}(t)$ are bounded and $\left(-F(t) \psi_{k}(l)+\int_{0}^{l} f \psi_{k} d x\right) \in$ $L_{2}[0, T]$. Thus the Cauchy problem has a unique solution $c_{k}^{N} \in C^{1}(0, T)$ for every $N$ and all approximations (20) are defined.

Next, we need a priori estimates to pass to the limit as $N \rightarrow \infty$.

Multiplying (21) by $\psi_{k}(x)$, summing from $k=0$ to $k=N$ and integrating with respect to $t$ from 0 to $\tau<T$, we obtain

$$
\begin{aligned}
& \frac{1}{2} \int_{0}^{l}\left(u^{N}\right)^{2} d x+\int_{Q_{\tau}}\left(u_{x}^{N}\right)^{2} d x d t=\frac{1}{2} \int_{0}^{l}\left(u^{N}(x, 0)\right)^{2} d x+\int_{Q_{\tau}} c\left(u^{N}\right)^{2} d x d t+ \\
& +\int_{Q_{\tau}} f u^{N} d x d t+\int_{Q_{\tau}} H(x) u_{x}^{N}(x, t) u^{N}(l, t) d x d t+\int_{0}^{\tau} F(t) u^{N}(l, t) d t .
\end{aligned}
$$

Aplying $\varepsilon$ - inequality and (15) to the terms of the right-hand side, we have

$$
\begin{aligned}
& \frac{1}{2} \int_{0}^{l}\left(u^{N}\right)^{2} d x+\frac{1}{2} \int_{Q_{\tau}}\left(u_{x}^{N}\right)^{2} d x d t \leq \frac{1}{2}\left(1+2 c_{1}+a(\varepsilon)+3 H_{0} a\left(\varepsilon_{1}\right)\right) \int_{Q_{\tau}}\left(u^{N}\right)^{2} d x d t+ \\
& +\frac{1}{2} \int_{0}^{l}\left(u^{N}\right)^{2}(x, 0) d x+\frac{1}{2} \int_{Q_{\tau}} f^{2} d x d t+\frac{1}{2} \int_{0}^{\tau} F^{2} d x d t
\end{aligned}
$$


where $H_{0}=T \int_{0}^{l} H^{2}(x) d x$ and $\varepsilon>0$ is an arbitrary constant.

Denote $m=1+2 c_{1}+a(\varepsilon)+3 H_{0} a\left(\varepsilon_{1}\right), G(\tau)=\int_{0}^{l}\left(u^{N}\right)^{2}(x, 0) d x+\int_{Q_{\tau}} f^{2} d x d t+\int_{0}^{\tau} F^{2} d t$ and obtain from (25) that

$\int_{0}^{l}\left(u^{N}\right)^{2}(x, \tau) d x \leq m \int_{0}^{\tau} \int_{0}^{l}\left(u^{N}\right)^{2} d x d t+G(\tau)$.

By Gronwall's lemma, we conclude

$\int_{0}^{\tau} \int_{0}^{l}\left(u^{N}\right)^{2} d x d t \leq \frac{G(\tau)}{m}\left(e^{m \tau}-1\right)$.

From (25) and (27) it follows that

$\int_{Q_{T}}\left(u_{x}^{N}\right)^{2} d x d t \leq G(T) e^{m T}$

And hence,

$\left\|u^{N}\right\|_{W_{2}^{1,0}\left(Q_{T}\right)} \leq M$,

where $M>0$ and does not depend on $N$.

Note that $W_{2}^{1,0}\left(Q_{T}\right)$ is Hilbert space. Therefore, because of (29), we can extract from $\left\{u^{N}\right\}$ a subsequence that convergence weakly in $W_{2}^{1,0}\left(Q_{T}\right)$ to some function $u(x, t) \in W_{2}^{1,0}\left(Q_{T}\right)$. We need only to show that this limit function is a required generalized solution.

To show that (9) is valid we multiply (21) by absolutely continuous functions $d_{m}(t), d_{m}(T)=0$ and $d_{m}^{\prime}(t) \in$ $L_{2}(0, T)$, take the sum from $m=1$ to $m=N$ and integrate with respect to $t$ from 0 to $T$. We obtain the following equality

$$
\begin{aligned}
& \int_{Q_{T}}\left(-u^{N_{k}} \Phi_{t}^{N}+u_{x}^{N_{k}} \Phi_{x}^{N}-c u^{N_{k}} \Phi^{N}\right) d x d t=\int_{Q_{T}} f \Phi^{N} d x d t+\int_{0}^{l} \varphi(x) \Phi^{N}(x, 0) d x+\int_{0}^{T} F(t) \Phi^{N}(l, t) d t+ \\
& +\int_{0}^{T} \int_{0}^{l} H(x) u_{x}^{N_{k}}(x, t) d x \Phi^{N}(l, t) d t,
\end{aligned}
$$

where $\Phi^{N}(x, t)=\sum_{m=1}^{N} d_{m}(t) \psi_{m}(x)$.

Taking into account the convergence proved above one can pass to the limit in (30) as $k \rightarrow \infty$ for any fixed $\Phi^{N}(x, t)$ and obtain

$$
\begin{aligned}
& \int_{Q_{T}}\left(-u \Phi_{t}^{N}+u_{x} \Phi_{x}^{N}-c u \Phi^{N}\right) d x d t=\int_{0}^{l} \varphi(x) \Phi^{N}(x, 0) d x+\int_{Q_{T}} f \Phi^{N} d x d t+ \\
& +\int_{0}^{T}\left(\int_{0}^{l} H(x) u_{x}(x, t) d x+F(t)\right) \Phi^{N}(l, t) d t .
\end{aligned}
$$

Note that the set of functions $\left\{\Phi^{N}\right\} \subset \bigcup_{N=1}^{\infty} \Phi^{N}$ is dense in $W_{2}^{1}\left(Q_{T}\right)[9]$. Let $\eta(x, t)$ be the limit function of $\left\{\Phi^{N}\right\}$ in $W_{2}^{1}\left(Q_{T}\right)$.

Consider the last term in the right-hand side of (31). We shall show that

$$
\lim _{N \rightarrow \infty} \int_{0}^{T}\left(\int_{0}^{l} H(x) u_{x}(x, t) d x+F(t)\right) \Phi^{N}(l, t) d t=\int_{0}^{T}\left(\int_{0}^{l} H(x) u_{x}(x, t) d x+F(t)\right) \eta(l, t) d t .
$$




$$
\begin{aligned}
& \text { Denote } \int_{0}^{l} H(x) u_{x}(x, t) d x+F(t)=S(t) \text {. Then } \\
& \left|\int_{0}^{T} S(t)\left(\Phi^{N}(l, t)-\eta(l, t)\right) d t\right| \leq \int_{0}^{T}|S(t)|\left|\left(\Phi^{N}(l, t)-\eta(l, t)\right)\right| d t \\
& \text { and }|S(t)|=\left|\int_{0}^{l} H(x) u_{x}(x, t) d x+F(t)\right| \leq\left|\int_{0}^{l} H(x) u_{x}(x, t) d x\right|+|F(t)| .
\end{aligned}
$$

As the estimate $(28)$ implies that $\int_{0}^{l} u_{x}^{2} d x \leq\left(G^{\prime}(T)+m G(T)\right) e^{m T}$, so

$$
\left(\int_{0}^{l} H(x) u_{x}(x, t) d x\right)^{2} \leq \int_{0}^{l} H^{2}(x) d x \int_{0}^{l} u_{x}^{2}(x, t) d x \leq H_{0}\left(G^{\prime}(T)+m G(T)\right) e^{m T} .
$$

Taking the square root of both sides in (34) we get that

$$
\left|\int_{0}^{l} H(x) u_{x}(x, t) d x\right| \leq\left[H_{0}\left(G^{\prime}(T)+m G(T)\right)\right]^{1 / 2} e^{m T / 2} .
$$

Moreover, $|F(t)| \leq \frac{\left|E^{\prime}\right|+c_{1}|E|+\left|f_{1}\right|}{|K(l)|},\left|f_{1}(t)\right|=\left|\int_{0}^{l} K f d x\right|$. And hence, $|S(t)| \leq A$, where $A$ depends on $l, T, K, c, f, E$.

Now consider the term $\int_{0}^{T}\left|\left(\Phi^{N}(l, t)-\eta(l, t)\right)\right| d t$. It is easy to see that

$$
\left(\int_{0}^{T}\left|\left(\Phi^{N}(l, t)-\eta(l, t)\right)\right| d t\right)^{2} \leq C^{2}\left(\int_{0}^{T} \int_{0}^{l}\left|\left(\Phi^{N}(x, t)-\eta(x, t)\right)_{x}\right| d x d t+\int_{0}^{T} \int_{0}^{l}\left|\left(\Phi^{N}(x, t)-\eta(x, t)\right)\right| d x d t\right)^{2} .
$$

This inequality implies that

$$
\left(\int_{0}^{T}\left|\left(\Phi^{N}(l, t)-\eta(l, t)\right)\right| d t\right)^{2} \leq 2 l T \int_{0}^{T} \int_{0}^{l}\left(\left(\left(\Phi^{N}-\eta\right)_{x}\right)^{2}+\left(\left(\Phi^{N}-\eta\right)\right)^{2}\right) d x d t \rightarrow 0 \quad \text { as } N \rightarrow \infty .
$$

Therefore (32) holds and the limit relation is fulfilled for every function $\eta \in W_{2}^{1}\left(Q_{T}\right)$, and hence, $u$ is the required solution of the problem (1)- (3), (6).

\section{Conclusion}

In this work we apply one of the possible methods of analysis of nonlocal problems with integral conditions of the first kind. Nonlocal problems with these type conditions are of considerable interest as the operator equation that corresponds the first type condition is ill-posed. We have shown that with certain conditions on a kernal and initial data the problem can be equivalent to the nonlocal problem with the second type integral condition. For the equivalent problem we introduce the notion of a generalized solution and prove its existence and uniqueness applying method of apriori estimates and Galerkin method.

\section{Acknowledgements}

The authour would like to thank Ludmila Pulkina for her constuctive comments. 


\section{References}

[1] J. R. Cannon, The solution of the heat equation subject to the specification of energy, Quart.Appl. Math., 21 (1963), no. $2,155-160$.

[2] R. Courant, D. Hilbert, Methods of Mathematical Physics, Interscience Publishers, inc.., New York, 1966.

[3] N.I. Ionkin, The solution of one boundary value problem of the theory of heat equation with non-classical bundary condition, Differen. uravn., 13 (1979), no.2, 294-304.

[4] L. I. Kamynin, On a boundary problem in the theory of heat conduction with a nonclassical boundary conditions, $Z h$. Vychisl. Math. Math. Fiz., 4 (1964), no. 6, 1006-1024.

[5] A.I. Kozhanov, On one non-local boundary problem with variable coefficients for heat equation and Aller's equation, Differen. uravn., 6 (2004), 763-774.

[6] A.I. Kozhanov, L.S. Pulkina, Boundary value problems with integral boundary condition for multidimensional hyperbolic equations, Doklady Academii Nauk, 404 (2005), no. 5, 589-592.

[7] A.I. Kozhanov, L.S. Pulkina, On solvability boundary value problems with nonlocal integral type boundary conditions for multidimensional hyperbolic equations, Differential equations, 42 (2006), no. 9, 1166-1179.

[8] O.A. Ladyzhenskaya, Boundary value problems of mathematical physics, Science, Moscow, 1973.

[9] O.A. Ladyzhenskaya , V.A. Solonnikov, N.N. Uralceva, Linear and quasi-linear parabolic eqations, Science, Moscow, 1967.

[10] L.S. Pontryagin, Ordinary differential equations (4th edition), Scince, Moscow, 1974.

[11] L. S. Pulkina, Initial-Boundary Value Problem with a Nonlocal Boundary Condition for a Multidimensional Hyperbolic equation,Differential equations, 44 (2008), no 8, 1119-1125.

[12] L.S. Pulkina, A nonlocal problem with integral conditions for the hyperbolic equation, Nanosystems: physics, chemistry, mathematics, no.2(4) (2011), 61-70.

[13] L.S. Pulkina, A nonlocal problem for a hyperbolic equation with integral conditions of the I st kind with time-dependent kernels, Russian Mathematics (Iz. VUZ), 56 (2012), no.10, 26-37.

[14] L.S. Pulkina, Nonclassical equations of mathematical physics, Publishing House of the Institute of Mathematics , 231-239 (2005). 